\title{
Robotic donor hepatectomy: a niche advancement or the way forward? A perspective from the world's largest center
}

\author{
Mark Sturdevant ${ }^{1}$, Ahmed Zidan ${ }^{2,3}$, Dieter Broering ${ }^{2,3}$ \\ 1Department of Surgery, Division of Transplantation, University of Washington Medical Center, Seattle, WA 98195, USA. \\ ${ }^{2}$ Department of Liver and Small Bowel Transplantation \& HPB Surgery, Organ Transplant Center, King Faisal Specialist Hospital \\ and Research Center, Riyadh 11211, Saudi Arabia. \\ ${ }^{3}$ Al Faisal University, Riyadh 11211, Saudi Arabia.
}

Correspondence to: Dr. Dieter Broering, MD, PhD, F.E.B.S., F.A.C.S., Department of Liver and Small Bowel Transplantation \& HPB Surgery, Organ Transplant Center, King Faisal Specialist Hospital and Research Center, MBC 96, P.O. Box 3354, Riyadh 11211,

Saudi Arabia. E-mail: dbroering@kfshrc.edu.sa

How to cite this article: Sturdevant M, Zidan A, Broering D. Robotic donor hepatectomy: a niche advancement or the way forward? A perspective from the world's largest center. Hepatoma Res 2022;8:1. https://dx.doi.org/10.20517/2394-

5079.2021.111

Received: 17 Aug 2021 First Decision: 29 Sep 2021 Revised: 13 Oct 2021 Accepted: 27 Oct 2021 Published: 6 Jan 2022

Academic Editor: Brian Kim Poh Goh Copy Editor: Xi-Jun Chen Production Editor: Xi-Jun Chen

\begin{abstract}
The application of minimally invasive liver surgery (MILS) in the field of living donor hepatectomy has been exceedingly slow, and its impact is limited to a handful of centers worldwide. Widespread adoption has been primarily hampered by the technical limitations of laparoscopy, namely rigid instrumentation, suboptimal optics, and a seemingly steep learning curve. These deficiencies are magnified in the donor hepatectomy operation wherein the parenchyma and vasculature must be handled atraumatically to produce a pristine allograft fit for implantation. Donor safety concerns and medicolegal ramifications are also cited as impediments to MILS in donor surgery. In 2013, our institution embraced a purely laparoscopic approach to living donor left lateral sectionectomy, and it quickly became our default technique. However, with donor hemi-hepatectomy, we gravitated to the robotic surgical system as our preferred modality. Herein, we describe our experience with minimally invasive donor hepatectomy, which we now universally offer to all living donors. Our extensive familiarity with robotic donor hepatectomy will provide the reader with an instructive perspective on the attributes and merits of the robotic approach. With appropriate collaboration and proctorship, we believe that the robotic platform will actualize a more rapid and widespread adoption than that experienced with the purely laparoscopic technique.
\end{abstract}


Keywords: Robotic liver surgery, robotic living donor hepatectomy, minimally invasive donor hepatectomy, live donor liver transplant

\section{INTRODUCTION}

In the early-to-mid 1990s, living donor liver transplantation (LDLT) was primarily developed to ameliorate waitlist mortality which resulted from an insufficient deceased donor pool. In countries with restrictive views regarding brain death and deceased organ donation, LDLT rapidly became a high-volume and impactful procedure as it was the predominant avenue to liver transplant (LT) for patients with advanced liver disease. However, even the most ardent proponent of LDLT acknowledged some unease with the inherent violation of primum non nocere, which occurs to some extent in every person undergoing living donor hepatectomy. Mitigating the violation of "first, do no harm" was the impetus for the application of minimally invasive liver surgery (MILS) to the field of living liver donation. This led Soubrane et al. ${ }^{[1]}$ to complete the first laparoscopic donor left lateral sectionectomy (L-LLS) in 2002, but few early adopters followed in their footsteps. A full decade passed before reports emerged from Soubrane et al. ${ }^{[1]}$ in Paris, Rotellar et al. ${ }^{[2]}$ in Spain, and Han et al. ${ }^{[3]}$ in Korea confirming the feasibility of purely laparoscopic living donor right hepatectomy while Troisi et al. ${ }^{[4]}$ did the same in Ghent in left lobe donors. Currently, pure laparoscopic donor hepatectomy (PLDH) for full lobe donors has largely been limited to the high-volume centers in Korea, along with a few select institutions in India and the West. Specifically, in 2017, Suh et al..$^{[5]}$ published the first sizeable series of right lobe PLDHs at Seoul National University and now has the largest such experience in the world. The dissemination of the PLDH approach has been slow and painstaking; enthusiasm for the technique has also been tempered by reports of higher than expected major (grade $\geq 3$ ) complication rates, especially in an institution's early experience (i.e., during the learning curve ${ }^{[6,7]}$. However, the benefits afforded to donors are undeniable regarding abdominal wall trauma and disfigurement and improvements in postoperative pain profiles.

By 2011, Giulianotti et al. ${ }^{[8,9]}$ had developed extensive experience in robotic liver surgery and utilized this approach to carry out the first robotic donor hepatectomy $(\mathrm{RDH})$ when he procured a right lobe at the University of Illinois-Chicago via the da Vinci robotic system. The first highly influential series in RDH came from Wu and Chen et al. ${ }^{[10]}$ at National Taiwan University when they reported on 13 cases of RDH in 2016. Minimally invasive donor hepatectomy (MIDH) now had two viable technical platforms.

\section{OUR JOURNEY IN MINIMALLY INVASIVE DONOR HEPATECTOMY}

In 2013, the author (Broering D) began utilizing MILS to carry out L-LLS at the King Faisal Specialist Hospital in Riyadh, which by that time had become a high-volume center in pediatric LDLT. The initial experience was aided by two major elements: (1) his extensive experience in split liver and open donor hepatectomy surgery; and (2) initial on-site collaboration with an experienced laparoscopic liver surgeon (Troisi R). By 2015, the purely laparoscopic technique had become our default and has been offered universally to left lateral section donors since that time. By April of 2018, we had performed more than onehundred L-LLS; this experience emboldened us to initiate an MIDH program for right and left lobe donors. Over an 8-month period, we performed twenty-six laparoscopic-assisted donor hepatectomies. The overall complication rate of $11.5 \%$ (no grade $\geq 3$ ) was gratifying, but the limitations of the laparoscopic technique (suboptimal optics, challenges in mobilizing the large right lobe, imprecise hilar plate transection, prohibitively long operative times) prompted us to pause and consider the alternative, namely the da Vinci Xi system (Intuitive Surgical Inc., Sunnyvale, CA, USA). The attributes of the robotic platform are welldescribed but worth mentioning in the context of the donor hepatectomy operation: (1) steady, magnified $(10 \times)$ and high-resolution 3D visualization; (2) enhanced suturing capability with tremor filtrating 
articulating instrumentation; and (3) improved surgeon ergonomics with less fatigue especially in this lengthy operation. Taken in their totality, we felt that these inherent advantages would allow us to better emulate the open donor hepatectomy operation and potentially with a smoother learning curve.

Prior to our index RDH case in November of 2018, we formed a collaborative relationship in the interest of donor safety with $\mathrm{Wu}$ and Chen et al. ${ }^{[10]}$ at the National Taiwan University, who published the aforementioned sole series (13 right lobes) in robotic MIDH. Preparation prior to our initial cases consisted of: (1) an observational visit to National Taiwan University; (2) extensive work with the robotic simulator; and (3) several minor hepatobiliary cases (no major robotic resections were performed). The first RDH cases in Riyadh were performed under the proctorship of our colleagues from Taipei by utilizing the dual teaching console. With this approach, we were able to complete these maiden cases robotically and found the platform to be more capable in our hands compared to pure laparoscopy. Our foray into RDH was not without some trepidation. This primarily pertained to two major issues: (1) the introduction and use of the Harmonic scalpel as the primary parenchymal transection modality [in open donor surgery, we use cavitron ultrasonic surgical aspiration (CUSA)]; and (2) duct transection without the aid of conventional cholangiography (a mandatory step in our open donor hepatectomies). A detailed description of our RDH technique has been published previously for both left lateral sectionectomy and full right/left lobe donor hepatectomies ${ }^{[11]}$.

From November 2018 to June 2021, the same senior console surgeon (Broering D) and bedside surgical team completed 356 robotic donor hepatectomies consisting of 158 right lobes, 75 left lobes, and 123 left lateral sectionectomies. This represents the largest series of RDH in the world cumulatively and for each living donor sub-type. There have been no donor mortalities, only 2 open conversions, and the overall complication rate for all donor types is just under $6 \%$ (no class $\geq 3$ complications). We specifically analyzed our early right lobe $\mathrm{RDH}$ experience by comparing our initial 35 robotic right lobe donors with a historical cohort of 70 open hepatectomy donors (via propensity-score matching). The analysis revealed a significant decrease in pain, blood loss, and in-hospital stay, as well as a trend toward lower morbidity in favor of $\mathrm{RDH}^{[12]}$. Despite longer donor warm ischemia times (median $7 \mathrm{~min}$ ), recipient outcomes did not suffer. Furthermore, we have not observed higher recipient bile duct issues, as has been noted in some laparoscopic MIDH series. Despite being a leading center in laparoscopic living donor left lateral sectionectomy, we have completely abandoned that technique in favor of the robotic platform and have been exceedingly pleased with the results for both the donors and recipients ${ }^{[13]}$.

\section{ROBOTIC DONOR HEPATECTOMY: THE FUTURE IS NOW}

\section{The King Faisal perspective}

Liver transplantation evolved from a mere technical marvel to one of the most transformative medical procedures of the 20th century because it became feasible and adoptable by the surgical masses. Pilgrimages to Pittsburgh and other pioneering centers in the 1980 s allowed LT to reach its level of greatness because it became an attainable, almost ubiquitous innovation. It has flourished and disseminated throughout the world and reaches most people privileged to have modern health care. It has been 20 years since the first laparoscopic donor hepatectomy was completed in Paris, but it remains a niche operation performed at a handful of institutions worldwide. We feel that the robotic platform has several attributes that piqued our interest initially, and now after $>350$ cases, they have solidified RDH as our preferred MIDH approach. This extensive experience positions us to offer some instructive guidance on how to utilize this modality and to provide perspective on its potential to be an impactful advancement in the field of liver donor surgery. 


\section{Prerequisites for a robotic donor hepatectomy program}

Thorough, thoughtful preparation is imperative for the successful implementation of an $\mathrm{RDH}$ program, and the following components are of paramount importance:

(1) The console surgeon should have extensive expertise in open donor hepatectomy. An experienced open living donor and split liver surgeon (our senior surgeon had completed $>500$ open donor hepatectomies) has the ideal technical background and skill set for RDH. The requisite robotic skills, of course, must be acquired and can be done in a relatively expeditious time course with a combination of simulation and completion of minor hepatobiliary and general surgical operations. Collaborating with a surgeon with robotic expertise during all phases of program development (training, actual cases) is strongly recommended.

(2) Surgical team: variability in the composition of the bedside surgical team (surgeons and scrub assistants) should be little to none. Bedside maneuvers, including port placement, instrument exchanges, and preparation of the extraction site, must be performed flawlessly. Significant time savings will result from a streamlined team that has developed harmony through repetition. Specifically, the entire team must be wellversed in troubleshooting a malfunctioning robotic system and must be able to rapidly undock and gain open access in emergent situations.

(3) Collaborators: prior to initiating our program, our senior surgeon (Broering D) observed numerous robotic cases at Taiwan National University (Wu CY, Chen PD), and they joined us in Riyadh for our first three RDHs. Successes in both our laparoscopic and robotic MIDH endeavors have come in no small part due to our high regard for collaboration and proctorship, and its importance cannot be overstated. Proctorship from an expert RDH surgeon will be a very important ingredient for initial success in an $\mathrm{RDH}$ $\operatorname{program}^{[14]}$.

(4) Liver donor selection: initially, we prioritized liver donors with conventional right lobe anatomy and a graft size $<800 \mathrm{~g}$. This was still more liberal than several of the laparoscopic donor hepatectomy centers who focused on right lobe grafts $<600 \mathrm{~g}$ in their initial experience. Preparing multiple cases in a short period of time is advantageous to smoothing out the learning curve. As experience and confidence grew, we rapidly liberalized our anatomic criteria for $\mathrm{RDH}$ donors, and it is now universally offered to all donors regardless of their anatomic complexity or body habitus.

(5) Institutional support: the da Vinci Xi platform is currently our preferred robotic platform, and access to a dual console is imperative for proctorship and training. Your institution must be prepared to aid the RDH program with ongoing financial support to remain relevant and up to date in this dynamic field.

\section{Overcoming perceived technical impediments to robotic donor hepatectomy}

The overriding goal for any minimally invasive modality is to allow a surgeon to perform the rehearsed steps of open surgery within the confines of a closed abdomen. It is our contention, after utilizing both platforms, that the robotic system is closer to realizing that aspiration. By capitalizing on the aforementioned technical innovations [superior optics, articulated instrumentation, tremor-free and precise suturing, real-time indocyanine green (ICG) Firefly], one can create an operation that closely emulates open donor hepatectomy.

Right lobe mobilization: accessing the postero-superior hepatic segments and atraumatically mobilizing the bulky right lobe has historically been a daunting challenge in the laparoscopic approach. The robotic 
platform allows the bedside (retracting left with the gallbladder) and console surgeons (stepwise retraction with a prograsper via arm 4) to work in a coordinated effort to accomplish this feat. Likewise, dissection and control of the short hepatic veins is accomplished exactly as one would do in the open procedure but with the advantage of a magnified field. Titanium clips (Challenger clips, Aesculap, Germany) are placed on the graft side and, to date, have had an excellent safety record. The importance of robotic suturing is highlighted in this step as all large direct hepatic veins (including the hepatocaval ligament) can be secured at the level of the inferior vena cava with suture ligature.

Hanging maneuver: the articulating dissector facilitates the development of the corridor between the right and middle hepatic veins, and a 10-Fr Foley catheter is inserted. With this technique, proponents of the hanging maneuver need not deviate from their standard open donor hepatectomy technique [Figure 1].

Parenchymal transection: changing our parenchymal transection modality (CUSA to robotic Harmonic scalpel) was our biggest reservation upon embarking on our $\mathrm{RDH}$ program, and this remains a concern for many contemplating the robotic platform. Proctorship via the dual console will markedly enhance the acquisition of this new skill. The magnified $3 \mathrm{D}$ view and pneumoperitoneum also allow the robotic surgeon to see and handle crossing veins in a remarkably bloodless field. Increasingly larger veins can be readily controlled by bipolar cautery, titanium clips, suture ligature, and endovascular staplers. Sutures placed into the liver on either side of the transection line are secured outside the skin to aid with retraction of the liver [Figure $2 \mathrm{~A}$ and $\mathrm{B}]$.

Dual-console training can play an exceedingly important role in alleviating the anxiety of utilizing a new technique such as the Harmonic scalpel. It is a unique feature of the robotic platform, which allows the proctoring surgeon to guide the operation in a very controlled and seamless manner. Ongoing research is now focused on how to most efficiently utilize the dual-console approach in teaching, training, and team building $^{[15]}[$ Figure 3].

Hilar plate transection: no cholangiogram, no problem. The magnified view afforded by the robotic platform and the real-time ICG (Firefly) mode facilitates precise bile duct identification. However, this step highlights the need for significant donor hepatectomy experience as the Firefly mode does not always provide a clear picture of duct anatomy. Identical to our open technique, we place a lacrimal probe (Bowman lacrimal probe AL1330, Microsurgical technology) into the duct after initial transection in order to confirm location and to identify the appropriate corridor for further hilar plate division ${ }^{[16]}[$ Figure $4 \mathrm{~A}$ and B]. Proficient remnant bile duct closure can be readily accomplished in $\mathrm{RDH}$ as reflected by our $2 \%$ bile leak rate (all grade 2 complication severity).

\section{ROBOTIC LIVER DONATION: TAKING THE SMOOTHER CURVE (LEARNING) TO WIDESPREAD ADOPTION}

The team from Seoul National University, with the largest laparoscopic MIDH series worldwide, estimates that it requires about 60 purely laparoscopic donor hepatectomies over 1 year in order to standardize the procedure $^{[17]}$. A lower volume center in Korea recommends that a center perform at least 70-75 cases of major laparoscopic hepatectomy before introducing laparoscopy into living donor surgery ${ }^{[18]}$. Even with extensive experience in living donor laparoscopic left lateral sectionectomy, we felt that the limitations of laparoscopy were major obstacles to mastering donor hemi-hepatectomy even after performing $>25$ cases with laparoscopic assistance. However, after recognizing the merits of the robotic platform in the fall of 2018, we rapidly acquired the ability to perform donor hepatectomy in a minimally invasive fashion. Since then, we have never looked back (to laparoscopy). We concur with our collaborators from Taiwan 


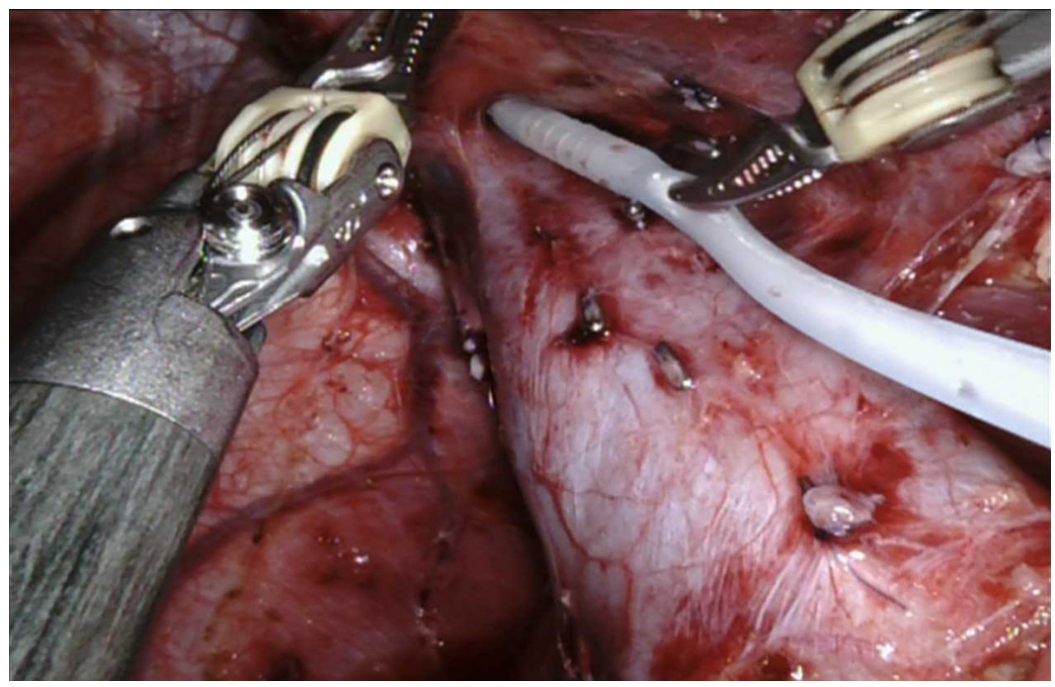

Figure 1. Creating the hanging maneuver: Foley catheter placement between the right and middle hepatic veins.
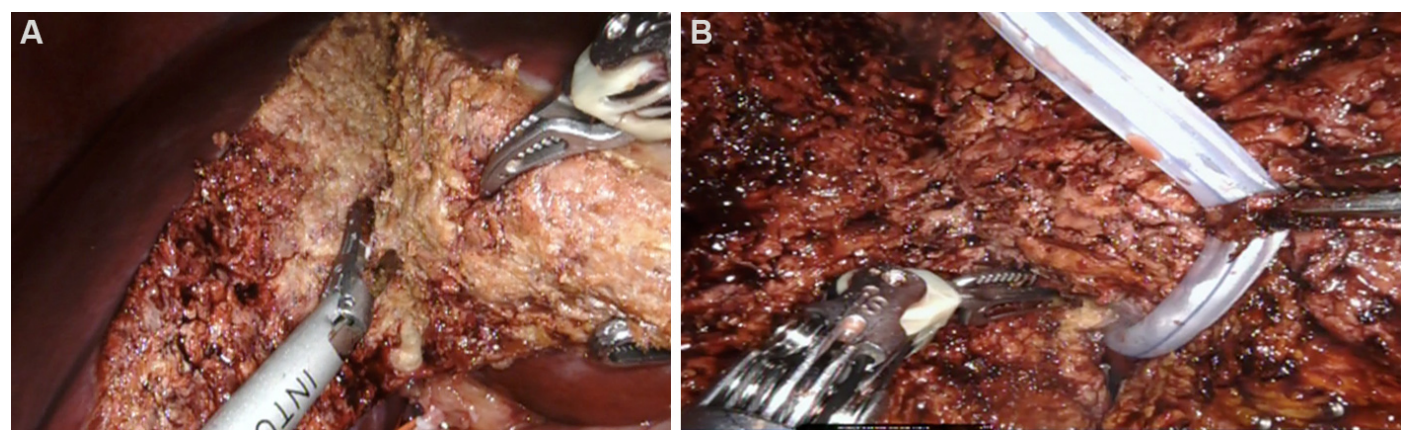

Figure 2. (A) Parenchymal transection with the Harmonic scalpel. (B) Utilizing the hanging maneuver in parenchymal transection.

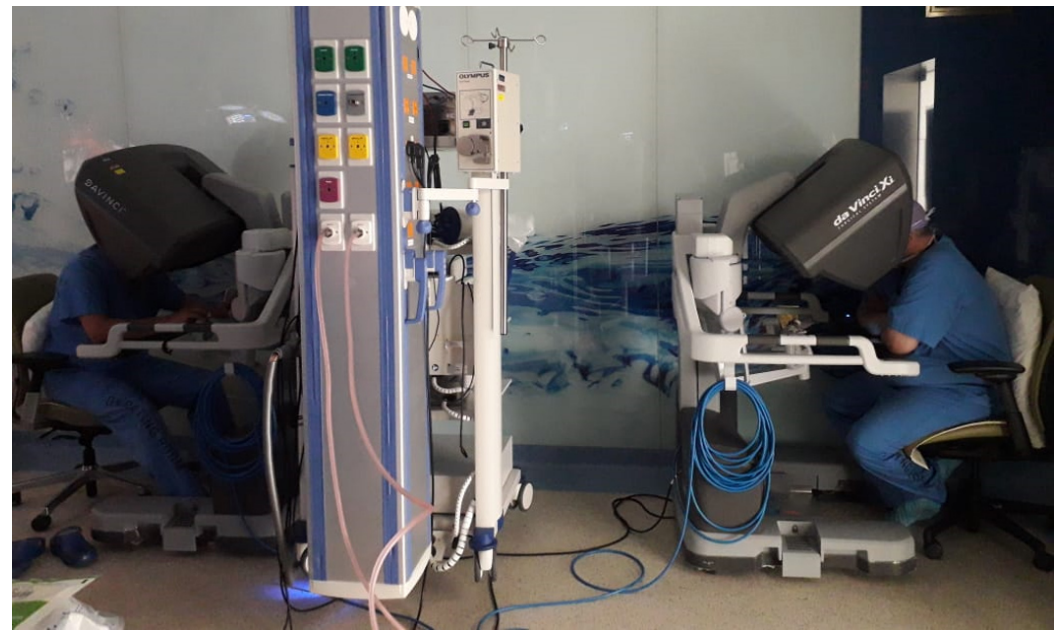

Figure 3. The dual console facilitates real-time proctorship and training.

University (Chen and $\mathrm{Wu}$ et al. ${ }^{[10]}$ ), who asserted years ago that the path to technical proficiency (i.e., learning curve) is far less steep and daunting with the robot compared to pure laparoscopy. To that point, in 

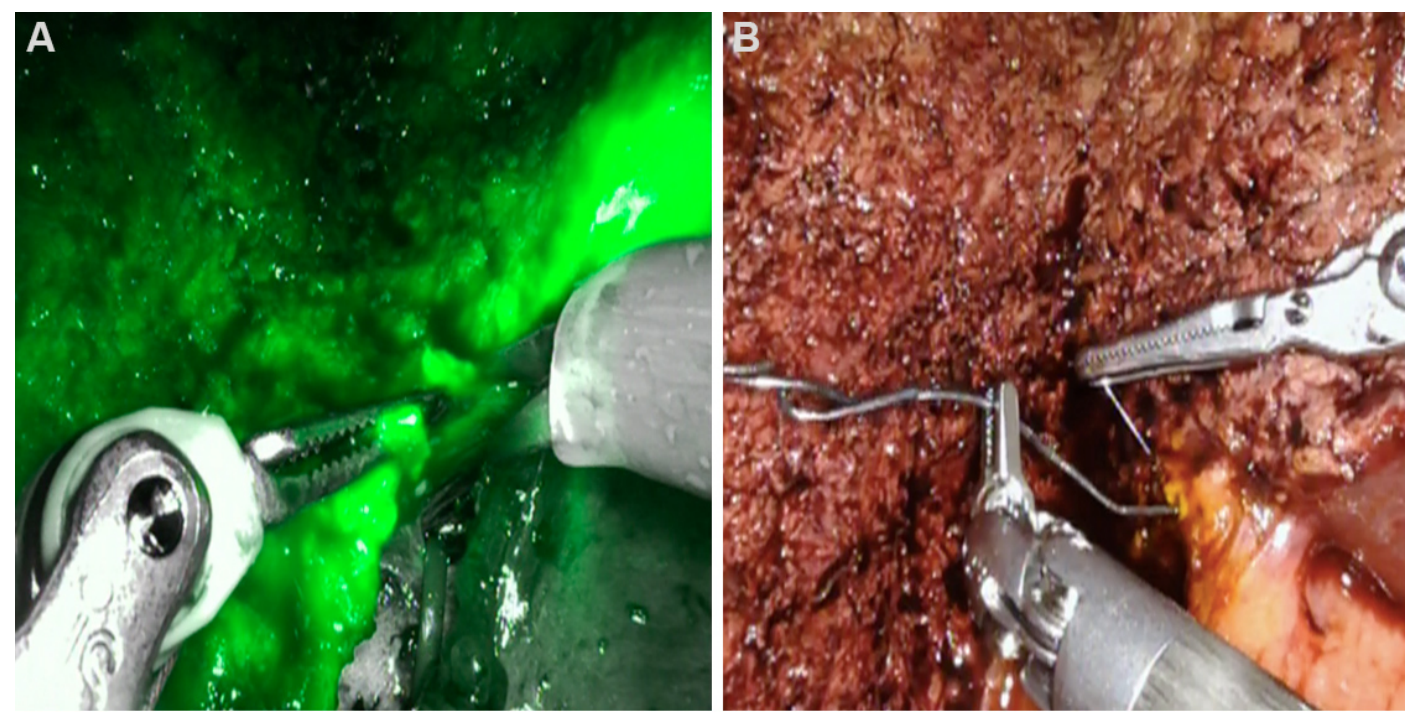

Figure 4. (A) Right hepatic duct transection under indocyanine green-guidance (Firefly). (B) Robotic probing of the transected remnant bile duct.

2019, 75\% (117 of 157) of our liver donations at King Faisal were completed with the robotic system. The next year in 2020 , just over a year after implementing the RDH program, we completed $92 \%$ of the 136 living liver donations by way of the robotic system. In that short period of time, full immersion into this technical platform was complete, and there were essentially no anatomic or body habitus contraindications for robotic utilization. This experience forms the basis of our profound enthusiasm and a hopeful prediction that we are at the threshold for global dissemination of minimally invasive surgery for liver donors. Multiple high-volume programs have been established; in addition to our group in Riyadh, Choi et al. ${ }^{[19]}$ and Rho et al. ${ }^{[20]}$ in Korea and Binoj et al. ${ }^{[21]}$ in India, have developed $\mathrm{RDH}$ expertise in countries highly dependent on LDLT. These success stories should embolden other primed institutions to pursue the robotic platform as their preferred MIDH modality. The major remaining barrier may reside in the cost of and access to the robotic platform itself, but with time we feel that these obstacles will become less ominous. Lastly, in the age of social media, liver donors everywhere will know the advantages of MIDH and will demand the safest and least morbid operation available. They will rightly ask for our best. With the proper combination of donor hepatectomy experience, acquisition of robotic skills, institutional support, and collaboration and proctorship, we feel that the robotic platform is the path forward.

\section{DECLARATIONS}

\section{Authors' contributions}

Performed all of the data acquisition: Zidan A, Broering D

Design, writing, and editing of the manuscript: Sturdevant M, Zidan A, Broering D

\section{Availability of data and materials}

Organ Transplant Center Registry King Faisal Specialist Hospital and Research Center, Riyadh, 11211 Saudi Arabia.

\section{Financial support and sponsorship}

None. 


\section{Conflicts of interest}

All authors declared that there are no conflicts of interest.

\section{Ethical approval and consent to participate}

Not applicable.

\section{Consent for publication}

Written informed consent was obtained for all images.

\section{Copyright}

(c) The Author(s) 2022.

\section{REFERENCES}

1. Soubrane O, Perdigao Cotta F, Scatton O. Pure laparoscopic right hepatectomy in a living donor. Am J Transplant 2013;13:2467-71. DOI

2. Rotellar F, Pardo F, Benito A, et al. Totally laparoscopic right-lobe hepatectomy for adult living donor liver transplantation: useful strategies to enhance safety. Am J Transplant 2013;13:3269-73. DOI PubMed

3. Han HS, Cho JY, Yoon YS, et al. Total laparoscopic living donor right hepatectomy. Surg Endosc 2015;29:184. DOI PubMed

4. Troisi RI, Wojcicki M, Tomassini F, et al. Pure laparoscopic full-left living donor hepatectomy for calculated small-for-size LDLT in adults: proof of concept. Am J Transplant 2013;13:2472-8. DOI PubMed

5. Suh KS, Hong SK, Lee KW, et al. Pure laparoscopic living donor hepatectomy: focus on 55 donors undergoing right hepatectomy. Am J Transplant 2018;18:434-43. DOI PubMed

6. Park J, Kwon DCH, Choi GS, et al. Safety and risk factors of pure laparoscopic living donor right hepatectomy: comparison to open technique in propensity score-matched analysis. Transplantation 2019;103:e308-16. DOI PubMed

7. Rhu J, Choi GS, Kim JM, Joh JW, Kwon CHD. Feasibility of total laparoscopic living donor right hepatectomy compared with open surgery: comprehensive review of 100 cases of the initial stage. J Hepatobiliary Pancreat Sci 2020;27:16-25. DOI PubMed

8. Giulianotti PC, Coratti A, Sbrana F, et al. Robotic liver surgery: results for 70 resections. Surgery 2011;149:29-39. DOI PubMed

9. Giulianotti PC, Tzvetanov I, Jeon H, et al. Robot-assisted right lobe donor hepatectomy. Transpl Int 2012;25:e5-9. DOI PubMed

10. Chen PD, Wu CY, Hu RH, et al. Robotic liver donor right hepatectomy: a pure, minimally invasive approach. Liver Transpl 2016;22:1509-18. DOI PubMed

11. Sturdevant ML, Zidan A, Broering D. Minimally-invasive donor hepatectomy at the dawn of a decade: can we pick up the pace? Dig Med Res 2020;3:58-58. DOI

12. Broering DC, Elsheikh Y, Alnemary Y, et al. Robotic versus open right lobe donor hepatectomy for adult living donor liver transplantation: a propensity score-matched analysis. Liver Transpl 2020;26:1455-64. DOI PubMed

13. Troisi RI, Elsheikh Y, Alnemary Y, et al. Safety and feasibility report of robotic-assisted left lateral sectionectomy for pediatric living donor liver transplantation: a comparative analysis of learning curves and mastery achieved with the laparoscopic approach. Transplantation 2021;105:1044-51. DOI PubMed

14. Broering DC, Berardi G, El Sheikh Y, Spagnoli A, Troisi RI. Learning curve under proctorship of pure laparoscopic living donor left lateral sectionectomy for pediatric transplantation. Ann Surg 2020;271:542-8. DOI PubMed

15. Cristofari H, Jung MK, Niclauss N, Toso C, Kloetzer L. Teaching and learning robotic surgery at the dual console: a video-based qualitative analysis. J Robot Surg 2021. DOI PubMed

16. Radtke A, Sotiropoulos GC, Molmenti EP, et al. Transhilar passage in right graft live donor liver transplantation: intrahilar anatomy and its impact on operative strategy. Am J Transplant 2012;12:718-27. DOI PubMed

17. Lee KW, Hong SK, Suh KS, et al. One hundred fifteen cases of pure laparoscopic living donor right hepatectomy at a single center. Transplantation 2018;102:1878-84. DOI PubMed

18. Lee B, Choi Y, Lee W, et al. Timing for introduction of total laparoscopic living donor right hepatectomy; initial experience based on the data of laparoscopic major hepatectomy. Transplantation 2021;105:1273-9. DOI PubMed

19. Choi GH, Chong JU, Han DH, Choi JS, Lee WJ. Robotic hepatectomy: the Korean experience and perspective. Hepatobiliary Surg Nutr 2017;6:230-8. DOI PubMed PMC

20. Rho SY, Lee JG, Joo DJ, et al. Outcomes of robotic living donor right hepatectomy from 52 consecutive cases: comparison with open and laparoscopy-assisted donor hepatectomy. Ann Surg 2020. DOI PubMed

21. Binoj ST, Mathew JS, Nair K, et al. 260 robotic donor right hepatectomy: is it just flaunting the scar? Gastroenterology 2020;158:S1263. DOI 